\title{
FINITE GROUPS WITH A STANDARD COMPONENT WHOSE CENTRALIZER HAS CYCLIC SYLOW 2-SUBGROUPS
}

\author{
LARRY FINKELSTEIN
}

\begin{abstract}
Let $G$ be a finite group with $O(G)=1, A$ a standard component of $G$ and $X$ the normal closure of $A$ in $G$. Furthermore, assume that $C(A)$ has cyclic Sylow 2-subgroups. Then conditions are given on $A$ which imply that either $X=A$ or $C(A)$ has Sylow 2-subgroups of order 2. These results are then applied to the cases where $A$ is isomorphic to $\cdot 0$ or $\hat{R} u$, the proper 2 -fold covering of the Rudvalis group.
\end{abstract}

1. Introduction. The combined work of Aschbacher and Seitz [2], [6] reduces the problem of classifying finite groups with a standard component $A$ of known type to the case where $C(A)$ has cyclic Sylow 2-subgroups. The object of this paper is to show that in many cases, the problem may be further reduced to the case where $C(A)$ has Sylow 2-subgroups of order 2. Our main results are proved in $\$ 2$ and are then applied in $\$ \S 3$ and 4 to the cases where $A$ is isomorphic to $\hat{R} u$ and $\cdot 0$, respectively, with $\hat{R} u$ being the full covering group of the Rudvallis group.

The author would like to thank Michael Aschbacher for improving the original version of Theorem 1 .

2. Main results.

THEOREM 1. Let $G$ be a finite group with $O(G)=1, A$ a standard component of $G$ and $X=\left\langle A^{G}\right\rangle$. Let $K=C(A)$ and $R \in \operatorname{Syl}_{2}(K)$ with $R \cong Z_{2^{n}}$. Then one of the following occurs.

(i) $X=A$.

(ii) $m(A)=1$ and $X$ is isomorphic to $L_{3}(q), q \equiv-1(\bmod 4), q \neq 3$, or $U_{3}(1), q \equiv 1(\bmod 4)$.

(iii) $R^{g} \leqslant N(A)$ for some $g \in G-N(A)$.

Proof. Assume that $X \neq A$ and let $g \in G-N(A)$ so that $Q=K^{g} \cap$ $N(A)$ has Sylow 2 subgroup $T$ of maximal order. If $|T|=|R|$, then (iii) holds. Hence assume that $|T|<|R|$. Since $K$ is tightly embedded in $G$ and 2-nilpotent by Burnside's transfer theorem, $N\left(R_{1}\right)$ covers $N(A) / O(N(A))$ for $1 \neq$ $R_{1} \leqslant R$. Thus if $\left|R_{1}\right|>|T|$ and $R_{1}^{h} \leqslant N\left(R_{1}\right)$ for some $h \in G$, then $h \in$ $N(A)$, hence $R_{1}^{h} \leqslant N_{K}\left(R_{1}\right)=R N_{O(K)}\left(R_{1}\right)$ which forces $R_{1}^{h}=R_{1}$ and shows that $R_{1}$ is weakly closed in $N\left(R_{1}\right)$ with respect to $G$. This implies that

Received by the editors March 9, 1976 and, in revised form, April 23, 1976.

AMS (MOS) subject classifications (1970). Primary 20DXX.

American Mathematical Society 1977 
$|T|>1$, for otherwise $\Omega_{1}(R)<Z(G)$ by the $Z^{*}$ theorem [10] and this contradicts $X \neq A$. Furthermore, we must then have $|R|>2$ in which case $X$ is simple and $G<\operatorname{Aut}(X)$ by Lemma 2.5 [4]. Let $\langle z\rangle=\Omega_{1}(R)$. If $|T|=2$ and $R_{1}$ is the unique $Z_{4}$ subgroup of $R$, then by [5, 2.5], $\left\langle z^{G}\right\rangle$ has dihedral or semidihedral Sylow 2-subgroups. Since $\left\langle z^{G}\right\rangle \geqslant X$ and $[z, A]=1$, it then follows that $z \in A$. Therefore $A$ has 2 rank 1 and $\left\langle z^{G}\right\rangle=X$. By the results of [1], $X$ must be isomorphic to $L_{3}(q), q \equiv-1(\bmod 4), q \neq 3$ or $U_{3}(q), q \equiv 1$ (mod 4), hence (ii) holds.

We may now assume that $|T|>2$ and, in addition, that $T$ normalizes $R$. By [3, Theorem 2], $C_{R}(T)=N_{R}(T) \cong T$, hence our assumption that $|T|<$ $|R|$ gives $C_{R}(T)<R$. Set $U=C_{R}(T)$ and let $U \leqslant V \leqslant R$ with $[V: U]=2$. Now $U T \triangleleft V T$ and $N_{V T}(T)=N_{V T}\left(\Omega_{1}(T)\right)$, so for $v \in V-U, U T=T \times$ $T^{v}$. This implies that $v$ inverts $[v, T]$ and since $V \triangleleft V T$, $v$ centralizes $[v, T]$ as well. But $[v, T] \cong T$ and a contradiction is established.

The main result of this paper concerns quasisimple groups which satisfy

Hypothesis I. $A$ is a quasisimple group such that $Z(A)$ has a cyclic Sylow 2-subgroup. Let $\bar{A}=A / Z(A), \bar{A} \leqslant \bar{L} \leqslant \operatorname{Aut}(\bar{A})$ and $\bar{t}$ an involution of $\bar{L}$. Then $C_{\bar{L}}(\bar{t}) / O\left(C_{\bar{L}}(\bar{t})\right)$ has no $Z_{4}$ or $Z_{2} \times Z_{4}$ normal subgroup.

THEOREM 2. Let $G$ be a finite group with $O(G)=1, A$ a standard component of $G$ satisfying Hypothesis I and $X=\left\langle A^{G}\right\rangle$. Assume that $K=C(A)$ has a cyclic Sylow 2-subgroup. Then either $X=A$ or $|K|_{2}=2$.

Proof. Assume that $X \neq A$ and $K$ has a Sylow 2-subgroup $R \cong Z_{2^{n}}$, $n>1$. If $\left|R^{8} \cap N(A)\right|<|R|$ for all $g \in G-N(A)$, then by Theorem 1(ii), $A \cong \mathrm{SL}_{2}(q), q$ odd, $q \neq 3$. But then $A$ does not satisfy Hypothesis I, hence by Theorem 1(iii), there exists $g \in G-N(A)$ such that $T=R^{g}$ is a Sylow 2-subgroup of $Q=K^{g} \cap N(A)$. It is easy to see that $Q$ is tightly embedded in $L=Q A$. In fact, if $Q \cap Q^{a}$ has even order for $a \in A$, then so does $K^{g} \cap K^{g a}$, hence $K^{g}=K^{g a}$. This in turn forces $Q^{a}=\left(K^{g} \cap N(A)\right)^{a}=K^{g}$ $\cap N(A)=Q$ as required. Let $\bar{L}=L / L \cap K$ and $\langle t\rangle=\Omega_{1}(T)$. Then $\bar{A} \leqslant \bar{L}$ $\leqslant \operatorname{Aut}(\bar{A})$ and $\bar{t}$ is an involution of $\bar{L}$.

Since $Q$ is tightly embedded in $L, C_{L}(t)$ normalizes $Q$ and this implies that $\overline{C_{L}(t)}$ normalizes $\bar{Q}=O(\bar{Q}) \bar{T}$. Let $\bar{C}=C_{\bar{L}}(\bar{t})$. We claim that $\bar{C}=\overline{C_{L}(t)}$ except when $L \cap K$ has an involution $z$ with $z \sim z t$ in $L$ in which case [ $\bar{C}$ : $\left.\overline{C_{L}(t)}\right]=2$. In order to prove this it suffices to show that $t$ centralizes a Sylow 2-subgroup $R_{0}$ of $L \cap K$. Without loss, we may assume that $R_{0} \leqslant R$ and $T$ normalizes $R$. But then $N_{R}(T) \cong T$ by [3, Theorem 2] whereupon [ $\left.R, T\right]=1$. Let $T_{1}$ be the unique $Z_{4}$ subgroup of $T$. Observe that $\left[\bar{C}: \overline{C_{L}(t)}\right] \leqslant 2$ implies that $O(\bar{C})=O\left(\overline{C_{L}(t)}\right)$. Since $\bar{Q} \cap \overline{C_{L}(t)}$ is a 2-nilpotent normal subgroup of $\overline{C_{L}(t)}$ with Sylow 2-subgroup $\bar{T}$, it then follows that $\bar{T} O(\bar{C}) \triangleleft \overline{C_{L}(t)}$. Hence $T_{1} O(\bar{C}) \triangleleft \overline{C_{L}(t)}$. By hypothesis, $\bar{C} / O(\bar{C})$ has no $Z_{4}$ or $Z_{2} \times Z_{4}$ normal subgroup, therefore we must have $\bar{C}=\left\langle\overline{C_{L}(t)}, \bar{\mu}\right\rangle$ where

$$
\left\langle\bar{T}_{1}, \bar{T}_{1}^{\bar{\mu}}\right\rangle O(\bar{C}) \triangleleft \bar{C} \text {. }
$$

Assuming that $\bar{T}_{1}^{\bar{\mu}}$ normalizes $\bar{T}_{1}$ and again invoking our hypothesis in 
conjunction with the fact that $\Omega_{1}\left(\bar{T}_{1}\right)=\Omega_{1}\left(\bar{T}_{1}^{\bar{\mu}}\right)=\langle\bar{t}\rangle$, we have $\left\langle\bar{T}_{1}, \bar{T}_{1}^{\bar{\mu}}\right\rangle \cong$ $Q_{8}$. A contradiction may now be established by noting that if $\bar{T}_{0}$ is the $Z_{4}$ subgroup of $\left\langle\bar{T}_{1}, \bar{T}_{1}^{\bar{\mu}}\right\rangle$ not equal to $\bar{T}_{1}$ or $\bar{T}_{1}^{\bar{\mu}}$, then $\bar{T}_{0} O(\bar{C}) \triangleleft \bar{C}$.

Theorem 2 extends [9, Proposition 5.2]. Many, but not all, of the sporadic groups satisfy Hypothesis I and, therefore, Theorem 2 should be useful in these standard component problems.

Suppose now that $G$ and $A$ satisfy the hypotheses of Theorem 2 except that $A$ does not satisfy Hypothesis I. Then the conclusion of Theorem 2 no longer holds. In fact, counterexamples exist when $G$ is isomorphic to $\operatorname{Aut}\left(F_{5}\right)$ [12] with $A$ isomorphic to the proper 2-fold covering of the Higman-Sims group $H S$ and $G$ is isomorphic to the O'Nan-Sims group [13] with $A$ isomorphic to a perfect central extension of $Z_{4}$ by $L_{3}(4)$. Note that $H S$ has an involution whose centralizer has a normal $Z_{4}$ subgroup whereas $L_{3}(4)$ has an involution whose centralizer has a normal $Z_{2} \times Z_{4}$ subgroup. At any rate, Theorem 1 may be helpful in these problems since it asserts that if $X \neq A$, then there exists $g \in G-N(A)$ such that $Q=K^{g} \cap N(A)$ has Sylow 2-subgroup $R^{g}$ and $Q$ is tightly embedded in $Q A$ with $R^{g}$ acting faithfully on $A$. One should then be able to use the structure of $A$ to determine $|R|$.

3. The case $A \cong \hat{R} u$. Let $R u$ be the simple group of order $2^{14} \cdot 3^{3} \cdot 5^{3} \cdot 7$. $13 \cdot 29$ whose existence was first proposed by Rudvalis and later confirmed by Conway and Wales [7]. The multiplier of $R u$ is known to have order 2 and the outer automorphism group is trivial (see [11]). The character tables of both $R u$ and $\hat{R} u$ have been computed by Frame and Rudvalis. The elements of 2 power order of $\hat{R} u$ are listed in the following table:

\section{TABLE I}

\begin{tabular}{clc}
$\hat{R} u$ Class & Centralizer Order & Squares \\
\hline \pm 1 & $2^{15} \cdot 3^{3} \cdot 5^{3} \cdot 7 \cdot 13 \cdot 29$ & 1 \\
$\pm 2 A$ & $2^{15} \cdot 3 \cdot 5$ & 1 \\
$4 A$ & $2^{10}$ & $-2 A$ \\
$\pm 4 B$ & $2^{10} \cdot 3 \cdot 5$ & $2 A$ \\
$4 C$ & $2^{9}$ & $2 A$ \\
$\pm 4 D$ & $2^{9} \cdot 3 \cdot 5$ & $-2 A$ \\
$4 E$ & $2^{8} \cdot 5 \cdot 7 \cdot 13$ & -1 \\
$\pm 8 A$ & $2^{7}$ & $4 A$ \\
$8 B$ & $2^{5}$ & $4 C$ \\
$\pm 8 C$ & $2^{6} \cdot 3 \cdot 5$ & $-4 B$ \\
$\pm 16 A$ & $2^{5}$ & $8 A$ \\
$\pm 16 B$ & $2^{5}$ & $-8 A$
\end{tabular}

Here $n A$ represents a class of elements of order $n,\langle-1\rangle$ is the center and $\pm n A$ denotes a double class of elements.

Lemma 3.1. Let $A \cong \hat{R}$. Then $A$ satisfies Hypothesis I. 
Proof. Let $\bar{A}=A / Z(A)$ and $\bar{t}$ be an involution of $\bar{A}$. It is clear from Table I that $\bar{A}$ has 2 classes of involutions, denoted by $2 A$ and $2 B$. Furthermore it is known that if $\bar{t} \in 2 A$ then $C_{\bar{A}}(\bar{t})$ is 2-constrained of order $2^{14} \cdot 3 \cdot 5$ with $C_{\bar{A}}(\bar{t}) / O_{2}\left(C_{\bar{A}}(\bar{t})\right) \cong S_{5}$ whereas if $\bar{t} \in 2 B$, then $C_{\bar{A}}(\bar{t}) \cong E_{4} \times S_{z}(8)$ (see [11]). Moreover, if $\bar{s}$ is an element of order 4 , then by Table $I,\left|C_{\bar{A}}(\bar{s})\right|_{2} \leqslant 2^{10}$. It now follows that in either case, $C_{\bar{A}}(\bar{t})$ has no $Z_{4}$ or $Z_{2} \times Z_{4}$ normal subgroup and $O\left(C_{\bar{A}}(\bar{t})\right)=1$. Thus $A$ satisfies Hypothesis $\mathrm{I}$ as required.

THEOREM 3. Let $G$ be a finite group with $O(G)=1, A$ a standard component of $G$ isomorphic to $R u$ and $X=\left\langle A^{G}\right\rangle$. Furthermore assume that $K=C(A)$ has cyclic Sylow 2-subgroups. Then $X=A$.

Proof. Assume by way of a contradiction that $X \neq A$. It then follows from Lemma 3.1 and Theorem 2 that $\langle z\rangle=Z(A) \in \operatorname{Syl}_{2}(K)$. Setting $L=C(z)$, we have $L=O(L) \times A$. It is clear from Table I that all involutions of $A-Z(A)$ are 4 th powers whereas $z$ is not. Therefore $z$ is isolated in $A$, hence in $L$ as well and we may conclude from the $Z^{*}$ theorem [10] that $z \in Z(G)$. This is incompatible with $X \neq A$ and the proof is completed.

4. The case $A \cong \cdot 0$. We begin by enumerating well-known properties of $\cdot 0$ (see [6]). .0 is a perfect group with center of order 2 and central quotient group isomorphic to $\cdot 1$. The outer automorphism group of $\cdot 1$ is trivial and $\cdot 0$ is a representation group for $\cdot 1$. Moreover $\cdot 1$ has 3 classes of involutions, denoted by $\left(2_{1}\right),\left(2_{2}\right)$ and $\left(2_{3}\right)$. If $r_{i} \in\left(2_{i}\right)$ and $L_{i}=C_{.1}\left(r_{i}\right)$, then $L_{1}$ is an extension of $Q_{8} * Q_{8} * Q_{8} * Q_{8}$ by $\Omega_{8}^{+}(2), L_{2}$ is an extension of $E_{2^{11}}$ by $\operatorname{Aut}\left(M_{12}\right)$ and $L_{3}$ is isomorphic to $E_{4} \times G_{2}(4)$ extended by an involution which acts as the field automorphism on $G_{2}(4)$ and together with $O_{2}\left(L_{3}\right)$ generates a $D_{8}$ subgroup. It is now evident that the following holds.

LFMMA 4.1. 1 satisfies Hypothesis I.

We can now prove the main result of this section.

THEOREM 4. Let $G$ be a finite group with $O(G)=1, A$ a standard component of $G$ isomorphic to $\cdot 0$ and $X=\left\langle A^{G}\right\rangle$. Assume that $K=C(A)$ has cyclic Sylow 2-subgroups. Then $X=A$.

Proof. Assume that $X \neq A$. It then follows from Lemma 4.1 and Theorem 2 that $\langle z\rangle=Z(A) \in \operatorname{Syl}_{2}(K)$. Thus $C(z)=A^{*} \times A$ where $A^{*}=O(C(z))$. Now finite groups in which the centralizer of an involution is isomorphic to . 0 have been classified [8]. In particular, such groups, modulo core, are isomorphic to $\cdot 0$. Moreover, the proof of [8] may be easily adapted to our present case and we conclude that $z \in Z^{*}(G)$, contradicting $X \neq A$.

\section{REFERENCES}

1. J. Alperin, R. Brauer and D. Gorenstein, Finite groups with quasi-dihedral and wreathed Sylow 2-subgroups, Trans. Amer. Math. Soc. 151 (1970), 1-261. MR 44 \# 1724. 
2. M. Aschbacher, A characterization of certain Chevalley groups and its application to component type groups, Proceedings of the Conference on Finite Groups, Academic Press, New York, 1976.

3. _ On finite groups of component type, Illinois J. Math. 19 (1975), 87-115. MR 51 \#13018.

4. _ Standard components of alternating type centralized by a 4-group (to appear).

5. - Finite groups in which the generalized fitting subgroup of the centralizer of some involution is symplectic but not extra special, Comm. Algebra 4 (1976), 595-616.

6. M. Aschbacher and G. Seitz, On groups with a standard component of known type (to appear).

7. J. Conway and D. Wales, Construction of the Rudvalis group of order 145,926,144,000, J. Algebra 27 (1974), 538-548. MR 49 \#400.

8. L. Finkelstein, Finite groups in which the centralizer of an involution is $\cdot 0$, J. Algebra 32 (1974), 173-177.

9. __ Finite groups with a standard component isomorphic to HJ or HJM, J. Algebra (to appear).

10. G. Glauberman, Central elements in core-free groups, J. Algebra 4 (1966), 403-420. MR 34 \#2681.

11. R. Griess, Schur multipliers of some sporadic simple groups, J. Algebra 32 (1974), 445-466.

12. K. Harada, On the simple group $F$ of order $2^{14} \cdot 3^{6} \cdot 5^{6} \cdot 7 \cdot 11 \cdot 19$, Proceedings of the Conference on Finite Groups, Academic Press, New York, 1976.

13. M. O'Nan, Some evidence for the existence of a new simple group, Proc. London Math. Soc. 32 (1976), 421-479.

Department of Mathematics, Wayne State University, Detroit, Michigan 48202 